\title{
Modeling and optimization of lipozyme RM IM-catalyzed esterification of medium- and long-chain triacyglycerols (MLCT) using response surface methodology
}

\begin{abstract}
Optimization conditions of Lipozyme RM IM lipase esterification of capric and stearic acids with glycerol for the production of medium- and long-chain triacyglycerols (MLCT) fat suitable for food applications such as margarine and shortening were investigated. Response surface methodology (RSM) was applied to model and optimize the reaction conditions, namely, the reaction time ( $8 \mathrm{i} ~ 24$ h), enzyme load (5ï 15 wt.\%), and fatty acids/glycerol ratio (3:1ï 4:1) and represented by $\mathrm{Ti}$, En, and $\mathrm{Sb}$, respectively. Best-fitting models were successfully established for both MLCT yield (R 2 䧃[D.9507) and residual FFA (R 2 困[0.9315) established by multiple regressions with backward elimination. Optimal reaction conditions were $13.6 \mathrm{i} 14.0 \mathrm{~h}$ for reaction time, 7.9ї $8.0 \mathrm{wt} \%$ for enzyme load, and 3:1 for fatty acids/glycerol molar ratio. Chi-square test showed that there were no significant (P®I05) differences between the observed and predicted values of both models. Refined MLCT fat blend had sufficient solid fat at room temperature and made it suitable to use as a hard stock in shortening and margarine production.
\end{abstract}

Keyword: Esterification; Medium- and long-chain triacyglycerols (MLCT); Response surface methodology (RSM); Reaction time; Enzyme load; Fatty acids/glycerol molar ratio; MLCT yield; Residual FFA 